\title{
Prevalencia de Fasciola hepatica y parásitos gastrointestinales en bovinos de la Región Amazonas, Perú
}

\section{Prevalence of Fasciola hepatica and gastrointestinal parasites in bovine of the Amazonas Region, Peru}

\author{
Deyvis Julon ${ }^{1}$, Víctor Puicón ${ }^{1,2}$, Amanda Chávez ${ }^{3}$, William Bardales ${ }^{1}$, Jhony \\ Gonzales ${ }^{1}$, Héctor Vásquez ${ }^{1}$, Jorge Maicelo ${ }^{1}$
}

\section{Resumen}

\begin{abstract}
El objetivo del presente estudio fue determinar la prevalencia de Fasciola hepatica y parásitos gastrointestinales en bovinos de cinco distritos de la Región Amazonas, Perú. Se colectaron 803 muestras fecales de bovinos entre octubre y diciembre de 2015 y se analizaron mediante la técnica coprológica de sedimentación espontánea. Los resultados indicaron una prevalencia de $F$. hepatica global de 59.5\%, y con mayor prevalencia en los distritos de Yambrasbamba y Florida, mientras que la prevalencia de parásitos gastrointestinales fue de $29.1 \%$, predominando en los distritos de Molinopampa y Huambo. Las variables procedencia, categoría y raza fueron factores que influyeron en el grado de infestación por F. hepatica y parásitos gastrointestinales $(\mathrm{p}<0.05)$, mientras que la variable sexo no fue significativa.
\end{abstract}

Palabras clave: Fasciola hepatica, parásitos gastrointestinales, sedimentación fecal

\section{Abstract}

The aim of this study was to determine the prevalence of Fasciola hepatica and gastrointestinal parasites in bovines from five districts of the Amazon Region, Peru. A total of 803 bovine faecal samples were collected between October and December 2015 and were analyzed by the coprological spontaneous sedimentation technique. The results indicated a global prevalence of $F$. hepatica of $59.5 \%$, with a higher prevalence in the districts of Yambrasbamba and Florida, while the prevalence of gastrointestinal

\footnotetext{
${ }^{1}$ Instituto de Investigación en Ganadería y Biotecnología, Universidad Nacional Toribio Rodríguez de Mendoza, Amazonas, Perú

${ }^{2}$ E-mail: victor puicon1704@hotmail.com

${ }^{3}$ Laboratorio de Microbiología y Parasitología Veterinaria, Universidad Nacional Mayor de San Marcos, Lima, Perú
}

Recibido: 9 de octubre de 2019

Aceptado para publicación: 28 de enero de 2020

Publicado: 31 de marzo de 2020 
parasites was $29.1 \%$, predominating in the districts of Molinopampa and Huambo. The variables origin, category and breed were factors that influenced the degree of infestation by $F$. hepatica and gastrointestinal parasites $(\mathrm{p}<0.05)$, while sex was not significant.

Key words: Fasciola hepatica, gastrointestinal parasites, faecal sedimentation

\section{INTRODUCCIÓN}

La Fasciola hepatica es un trematodo que causa una enfermedad zoonótica conocida como fasciolosis (Torgerson, 2013). Es de distribución mundial y afecta la salud de especies domésticas y silvestres, así como también al humano, generando pérdidas económicas significativas en el sector pecuario mundial y en la salud pública (Arroyo et al., 1981). La Organización Mundial de la Salud (OMS) ha estimado que existen 2.4 millones de personas infectadas con $F$. hepatica y unos 180 millones adicionales en riesgo de infección (Becerra, 2001).

López-Lemes et al. (2005) mencionan que 300 millones de bovinos y 250 millones de ovinos están en riesgo de ser afectados a nivel global, mientras que en el Perú se le considera como la segunda enfermedad parasitaría económicamente más importante en la ganadería, causando pérdidas que superan los 50 millones de dólares al año, solo en ganado vacuno (Espinoza et al., 2010). Es una parasitosis que se le encuentra distribuida en todo el país (Manrique y Cuadros, 2002; Páucar et al., 2010). En términos de salud pública, la fasciolasis humana se ha reportado en varios lugares del Perú, incluyendo la región de Amazonas, en tanto que en términos de fasciolasis bovina, no existen reportes previos en el ganado de esta zona.

La información generada en la presente investigación, tuvo como objetivo determinar la prevalencia de $F$. hepatica y parásitos gastrointestinales en bovinos de las principales zonas ganaderas de la región Amazonas, Perú, y evaluar la significancia de variables como procedencia, sexo, categoría y raza sobre la presencia parasitaria.

\section{Materiales y Métodos}

\section{Población y Área de Estudio}

La población animal estuvo conformada por ganado bovino con alto contenido genético de las razas Brown Swiss, Simmental, Angus, Holstein y Jersey, además del ganado criollo local, con edades que varían desde 6 meses hasta 4 años. Los animales estaban distribuidos en las cuencas ganaderas de Leyva, Ventilla y Pomacochas, correspondientes a los distritos de Huambo y Limabamba (provincia de Rodríguez de Mendoza), Molinopampa (provincia de Chachapoyas), Florida y Yambrasbamba (provincia de Bongará), respectivamente (Figura 1).

Estas zonas se encuentran en áreas de acción de las estaciones experimentales de la Universidad Nacional Toribio Rodríguez de Mendoza de Amazonas. Se encuentran localizadas a 1699-2335 msnm y presentan una precipitación pluvial anual de 738-1537 mm y una temperatura media anual de $16.5^{\circ} \mathrm{C}$.

Los animales se mantuvieron al pastoreo con diversas variedades de pastos naturales y cultivados. En las zonas de estudio se observan factores predisponentes a fasciolasis, debido a la proximidad de los ríos, lagos y lagunas. El muestreo parasitológico se llevó a cabo entre octubre y diciembre de 2015. Los animales muestreados no recibieron dosificación alguna en los 120 días previos a la realización del estudio. 


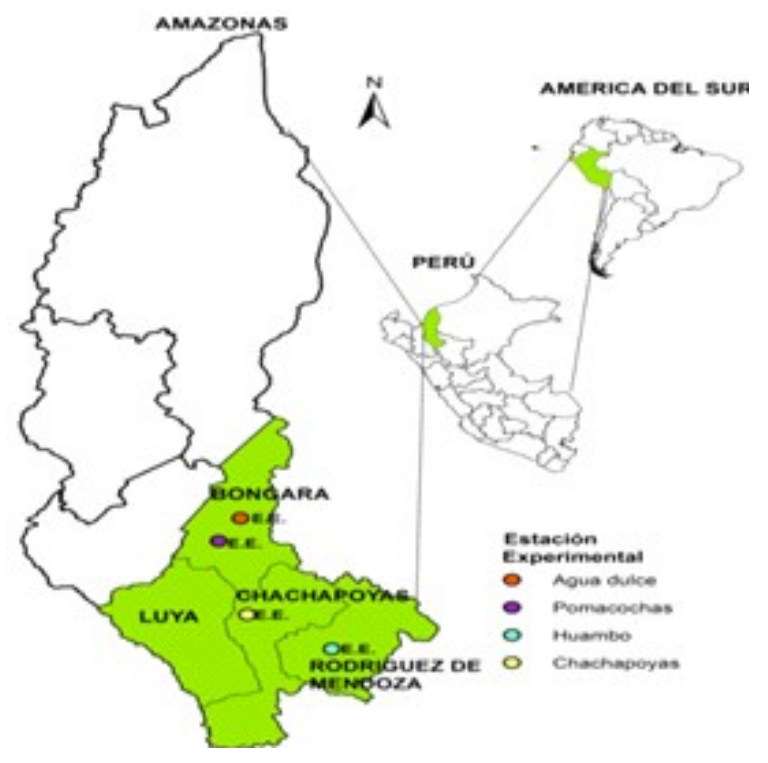

Figura 1. Mapa de ubicación del área de estudio

\section{Tamaño de Muestra}

En la zona de estudio se estimó una población de 29263 cabezas de ganado (INEI, 2012). Al no contar con estudios previos respecto a la prevalencia de fasiolasis en la zona, se optó por tomar la probabilidad de $0.68 \mathrm{de}$ hallar animales con parásitos gastrointestinales y F. hepatica (Colina et al., 2013), resultando un tamaño de muestra de 803 animales. El mecanismo de selección fue por muestreo estratificado con afijación proporcional para garantizar que todos los distritos de la región en estudio se encuentren debidamente representados en la muestra (Cuadro 1).

\section{Muestras y Técnica Coproparasitológica}

El muestreo coprológico se realizó en las primeras horas de la mañana. Se tomaron $20 \mathrm{~g}$ de heces del recto del animal y fueron almacenadas en bolsas de polietileno, que fueron trasladadas con refrigerantes y sin conservantes al Laboratorio de Parasitología de la Facultad de Medicina Veterinaria de la Universidad Nacional Mayor de San Marcos, Lima. Desde la toma de muestras fecales hasta su procesamiento transcurrieron entre dos a tres días por cada lote de muestras.

En el análisis de las muestras se empleó la técnica de sedimentación espontánea para identificar helmintos, incluyendo trematodos como Fasciola hepatica y Paramphistomum sp (Terashima et al., 2009). Debido a que el objetivo central de este estudio fue la evaluación de la presencia de $F$. hepatica, se estableció el criterio de considerar positivas aquellas muestras en las que se observó al menos un huevo típico de F. hepatica. El huevo de este parásito es de forma ovoide, color amarillo, con presencia de opérculo en un polo, y con medidas de 130 a $150 \times 63$ a $90 \mu \mathrm{m}$, el cual se diferencia del huevo de Paramphistomum sp, que es de color claro, cigoto localizado en la parte medial posterior y con medidas de 114 a 176 x 73 a $104 \mu \mathrm{m}$ (Rojas y Torrel, 2004). 
Cuadro 1. Tamaño de muestra de unidades pecuarias por área de influencia de los distritos del estudio

\begin{tabular}{|c|c|c|c|c|c|}
\hline Distritos & $\begin{array}{c}\text { Unidades } \\
\text { pecuarias } \\
\text { por } \\
\text { distrito } \\
\end{array}$ & $\begin{array}{l}\text { Población } \\
\text { por distrito }\end{array}$ & $\begin{array}{l}\text { Muestra } \\
\text { de } \\
\text { unidades } \\
\text { pecuarias }\end{array}$ & $\begin{array}{c}\text { Vacunos por } \\
\text { unidad } \\
\text { pecuaria }\end{array}$ & $\begin{array}{l}\text { Bovinos } \\
\text { por } \\
\text { distrito }\end{array}$ \\
\hline $\begin{array}{l}\text { Molinopampa (Prov. } \\
\text { Chachapoyas) }\end{array}$ & 4,133 & 7,921 & 20 & 12 & 237 \\
\hline Florida (Prov. Bongará) & 4,846 & 7,742 & 23 & 10 & 232 \\
\hline $\begin{array}{l}\text { Yambrasbamba (Prov. } \\
\text { Bongara) }\end{array}$ & 3,705 & 5,500 & 18 & 8 & 142 \\
\hline $\begin{array}{l}\text { Huambo (Prov. } \\
\text { Rodríguez de Mendoza) }\end{array}$ & 2,194 & 2,671 & 10 & 11 & 115 \\
\hline $\begin{array}{l}\text { Lima bamba (Prov. } \\
\text { Rodríguez de Mendoza) }\end{array}$ & 2,694 & 5,429 & 13 & 6 & 77 \\
\hline Total & 17,572 & 29,263 & 84 & 47 & 803 \\
\hline
\end{tabular}

\section{Variables de Estudio}

Se consideraron variables dependientes de estudio a la procedencia (Huambo, Limabamba, Molinopampa, Florida, Yambrasbamba), sexo (macho, hembra), categoría etaria (toro, vaca, torete, ternera, vaquillona, ternero) y raza (Holstein, Brown Swiss, Simmental, Angus, Jersey y criollo).

\section{Análisis Estadístico}

Se determinó la prevalencia de la enfermedad mediante la determinación del número de muestras fecales positivas. Los resultados se expresaron en tablas estratificadas para comparar el efecto de las variables procedencia, sexo, categoría y raza, bajo un nivel de significancia de 0.05 mediante análisis de varianza y prueba de comparaciones múltiples de Tukey en aquellos que se detectó diferencia estadística significativa. Se empleó el paquete estadístico SPSS 20.

\section{Resultados}

La prevalencia de Fasciola hepatica fue de 59.5\% (478/803) con mayor presencia en Florida y Yambrasbamba (85.3\%) y $79.6 \%$ ), distritos pertenecientes a la cuenca ganadera de Pomacochas (Cuadro 2). En el caso de los parásitos gastrointestinales, se encontró una prevalencia global de $29.1 \%$ (234/803), observándose una mayor prevalencia en el distrito de Molinopampa (50.6\%) $\mathrm{El}$ análisis de varianza indicó que existe diferencia significativa $(\mathrm{p}<0.05)$ según la procedencia del ganado bovino. Al aplicar la prueba de comparaciones múltiples de Tuckey resulta que los distritos de Florida y Yambrasbamba presentaron mayor prevalencia de $F$. hepatica, en tanto que los animales del distrito de Molinopampa tuvieron la mayor presencia de parásitos gastrointestinales (Cuadro 2). Por otro lado, no se encontró diferencia significativa debido al sexo de los animales (Cuadro 3). 
Cuadro 2. Prevalencia (\%) de Fasciola hepatica y parásitos gastrointestinales en bovinos de la región de Amazonas, Perú, mediante la técnica de sedimentación, según procedencia

\begin{tabular}{lccc}
\hline Distritos & $\begin{array}{c}\text { Bovinos } \\
(\mathrm{n})\end{array}$ & $\begin{array}{c}\text { F. hepatica } \\
(\%)\end{array}$ & $\begin{array}{c}\text { Parásitos } \\
\text { gastrointestinales } \\
(\%)\end{array}$ \\
\hline Huambo & 115 & $44.4^{\mathrm{b}}$ & $36.5^{\mathrm{b}}$ \\
Limabamba & 77 & $36.3^{\mathrm{b}}$ & $28.6^{\mathrm{b}}$ \\
Molinopampa & 237 & $37.1^{\mathrm{b}}$ & $50.6^{\mathrm{a}}$ \\
Florida & 232 & $85.3^{\mathrm{a}}$ & $12.5^{\mathrm{b}}$ \\
Yambrasbamba & 142 & $79.6^{\mathrm{a}}$ & $14.8^{\mathrm{b}}$ \\
\hline Total & 803 & 59.5 & 29.1 \\
\hline
\end{tabular}

a,b Superíndices diferentes dentro de cada columna y variable indican diferencia estadística $(p<0.05)$

Cuadro 3. Prevalencia (\%) de Fasciola hepatica y parásitos gastrointestinales en bovinos de la región de Amazonas, Perú, mediante la técnica de sedimentación, según sexo

\begin{tabular}{lccc}
\hline Sexo & $\begin{array}{c}\text { Bovinos } \\
(\mathrm{n})\end{array}$ & $\begin{array}{c}\text { F. hepatica } \\
(\%)\end{array}$ & $\begin{array}{c}\text { Parásitos } \\
\text { gastrointestinales (\%) }\end{array}$ \\
\hline Macho & 109 & 59.6 & 28.4 \\
Hembra & 694 & 59.5 & 29.2 \\
\hline Total & 803 & 59.5 & 29.1 \\
\hline
\end{tabular}

En el análisis de la variable categoría del ganado, se evidenció que el caso de $F$. hepatica, la prevalencia fue incrementándose conforme aumentaba la madurez del animal; mientras que para el caso de los parásitos gastrointestinales, los animales jóvenes fueron los más afectados (Cuadro $4 ; \mathrm{p}<0.05$ ).

Se observó una mayor presencia de $F$. hepatica en el ganado Simmental, Angus y Brown Swiss $(p<0.05)$, mientras que en el caso de los parásitos gastrointestinales, los animales de las razas Jersey y Holstein presentaron una mayor prevalencia $(\mathrm{p}<0.05$; Cuadro 5).

\section{Discusión}

Los resultados obtenidos revelan una prevalencia del $59.5 \%$ de $F$. hepatica en la región Amazonas, superior a lo hallado en otras zonas del país, como Ayacucho (35.9\%) y Junín (55.7\%) (Ticona et al., 2010; Castro et al., 2002), pero inferior a valores hallados en países vecinos como Colombia (90\%) (Wilches et al., 2009).

Las mayores prevalencias de fasciolasis se encontraron en los distritos de Florida (85.3\%), Yambrasbamba (79.6\%) y Huambo (44.4\%). Esto puede deberse a que en estos 
Cuadro 4. Prevalencia (\%) de Fasciola hepatica y parásitos gastrointestinales en bovinos de la región de Amazonas, Perú, mediante la técnica de sedimentación, según categoría animal

\begin{tabular}{lccc}
\hline Categoría animal & $\begin{array}{c}\text { Bovinos } \\
(\mathrm{n})\end{array}$ & $\begin{array}{c}\text { F. hepatica } \\
(\%)\end{array}$ & $\begin{array}{c}\text { Parásitos } \\
\text { gastrointestinales (\%) }\end{array}$ \\
\hline Toro & 47 & $70.2^{\mathrm{a}}$ & $17.0^{\mathrm{b}}$ \\
Vaca & 514 & $63.8^{\mathrm{a}}$ & $26.5^{\mathrm{b}}$ \\
Torete & 27 & $63.0^{\mathrm{a}}$ & $18.5^{\mathrm{b}}$ \\
Ternera & 115 & $42.6^{\mathrm{b}}$ & $39.1^{\mathrm{a}}$ \\
Vaquillona & 57 & $52.6^{\mathrm{b}}$ & $35.1^{\mathrm{a}}$ \\
Ternero & 43 & $48.8^{\mathrm{b}}$ & $46.5^{\mathrm{a}}$ \\
\hline Total & 803 & 59.5 & 29.1 \\
\hline
\end{tabular}

$a, b, c$ Superíndices diferentes dentro de cada columna y variable indican diferencia estadística $(p<0.05)$

Cuadro 5. Prevalencia (\%) de Fasciola hepatica y parásitos gastrointestinales en bovinos de la región de Amazonas, Perú, mediante la técnica de sedimentación, según el grupo racial

\begin{tabular}{lccc}
\hline Raza & $\begin{array}{c}\text { Bovinos } \\
(\mathrm{n})\end{array}$ & $\begin{array}{c}\text { F. hepatica } \\
(\%)\end{array}$ & $\begin{array}{c}\text { Parásitos } \\
\text { gastrointestinales } \\
(\%)\end{array}$ \\
\hline Brown Swiss & 347 & $60.8^{\mathrm{a}}$ & $31.1^{\mathrm{b}}$ \\
Simmental & 149 & $71.1^{\mathrm{a}}$ & $20.8^{\mathrm{b}}$ \\
Angus & 12 & $66.7^{\mathrm{a}}$ & $25.0^{\mathrm{b}}$ \\
Holstein & 68 & $51.5^{\mathrm{b}}$ & $39.7^{\mathrm{a}}$ \\
Jersey & 20 & $55.0^{\mathrm{b}}$ & $40.0^{\mathrm{a}}$ \\
Cruzado & 195 & $54.4^{\mathrm{b}}$ & $27.2^{\mathrm{b}}$ \\
\hline Total & 791 & 60.3 & 29.1 \\
\hline
\end{tabular}

${ }^{a, b}$ Superíndices diferentes dentro de cada columna y variable indican diferencia estadística $(p<0.05)$

distritos, las zonas ganaderas muestreadas estaban muy próximas a los ríos y lagunas, como es el caso de la laguna de Pomacochas en Florida, el Rio Imaza en Yambrasbamba y el río Leyva en Huambo, lo que genera la presencia cercana de riachuelos, arroyos, acequias pastizales húmedos y pantanos; además, la fasciolasis se encuentra favorecida por las condiciones epidemiológicas favora- bles de temperatura y humedad en estas zonas, las cuales son ideales para el desarrollo de los huevos y la liberación de miracidios, así como del desarrollo del hospedero intermediario durante todo el año (Leguía y Casas, 1999). Es en estas zonas donde, por lo regular, las precipitaciones pluviales son frecuentes, especialmente en la época de muestreo (inicio de la estación lluviosa) que 
favorece el potencial de infección F. hepatica en el ganado (Leguía y Casas, 1999; Olaechea, 2004).

La prevalencia de parásitos gastrointestinales fue de $29.1 \%$, con mayor presencia en los distritos de Molinopampa y Huambo. Esta frecuencia fue menor al 51.1\% reportado por Armijos (2013) en Ecuador y al 60.6\% encontrado por Rodríguez-Vivas et al. (2001) en México. Al respecto, es conocido que la infestación de los animales con parásitos gastrointestinales ocurre al ingerir larvas infectivas que se encuentran presentes en las pasturas o en el agua de lugares estancados (Bertucci y Goslino, 2014).

Los resultados muestran una mayor prevalencia de $F$. hepatica en animales adultos (vacas: $63.8 \%$ y toros $70.2 \%$ ), resultado similar a lo encontrado por Alpízar et al. (2013) en Costa Rica y Prepelitchi (2009) en Argentina. No obstante, cabe indicar que los terneros son más susceptibles a la acción patógena de $F$. hepática, pudiendo ocurrir muertes repentinas antes de la observación de signos clínicos, lo cual está en relación con el desarrollo de los mecanismos inmunes y del tejido conectivo hepático (Leguía y Casas, 1999). Por otro lado, la mayor prevalencia de parásitos gastrointestinales en animales jóvenes (terneras: $39.1 \%$; terneros: $46.5 \%$ ) es compatible con los reportes que indican una mayor susceptibilidad de animales jóvenes (Zárate, 2003), aunque Paredes (2014) afirma que la presencia de parásitos no está condicionada por la categoría del bovino.

El presente estudio muestra que la presencia de parásitos está condicionada por la raza de los bovinos, siendo los animales de las razas Simmental (71.1\%) y Brown Swiss $(60.8 \%)$ los más afectados por $F$. hepatica. Estos resultados fueron similares a lo reportado por Sanchis et al. (2011) quienes encontraron diferencia significativa en la prevalencia de este trematodo entre razas bovinas. Particular atención merece el resultado de los animales cruzados, que parecen ser los más resistentes a este parásito. Con respecto a parásitos gastrointestinales, las mayores prevalencias se encontraron en animales Jersey (40.0\%) y Hosltein (39.7\%); sin embargo, Oliveira et al. (2009), trabajando en Brasil con razas cárnicas no encontraron diferencias significativas entre razas.

\section{Conclusiones}

- La prevalencia de Fasciola hepatica en la región de Amazonas fue de 59.5\%, siendo mayor en los distritos de Yambrasbamba y Florida, mientras que la prevalencia de parásitos gastrointestinales fue de $29.1 \%$, predominando en los distritos de Molinopampa y Huambo.

- Las variables procedencia, categoría y raza fueron factores que influyeron en el grado de infestación por $F$. hepatica y parásitos gastrointestinales $(\mathrm{p}<0.05)$, mientras que la variable sexo no fue significativa.

\section{Literatura Citada}

1. Alpízar C, Bianque de Oliveira J, Jiménez AE, Hernández J, Berrocal A, Romero JJ. 2013. Fasciola hepatica en ganado bovino de carne en Siquirres y lesiones anatomo-histopatológicas de hígados bovinos decomisados en mataderos de Costa Rica. Agron Costar 37(2): 7-16.

2. Armijos NI. 2013. Prevalencia de parásitos gastrointestinales de bovinos que se sacrifican en el camal municipal de Santa Isabel. Tesis de Médico Veterinario. Cuenca: Univ de Cuenca. $160 \mathrm{p}$.

3. Arroyo R, Mora J, Molina S, Troper L, Amador A. 1981. Fascioliasis hepática humana en Costa Rica. Rev Costar Cienc Méd 2(1): 35-57.

4. Becerra W. 2001. Consideraciones sobre estrategias sostenibles para el control de Fasciola hepatica en Latinoamérica. Rev Col Cienc Pec 14: 28-35. 
5. Bertucci LO, Goslino MI. 2014. Estudio comparativo de los endoparásitos en bovinos Bonsmara-Hereford y Hereford puros en iguales condiciones de manejo. Tesis de Doctorado. Montevideo: Univ. de la Republica. 93 p.

6. Castro J, Yovera J, Colona E. 2002. Detección de coproantígenos de Fasciola hepatica en vacunos mediante un sandwich-ELISA. Rev Perú Parasitol 16(1): 10-13.

7. [INEI] Instituto Nacional de Estadística e Informática. 2012. IV Censo Nacional Agropecuario 2012. [Internet]. Disponible en: censos.inei.gob.pe/ Cenagro/redatam

8. Colina JC, Mendoza GA, Jara CA. 2013. Prevalencia e intensidad del parasitismo gastrointestinal por nematodos en bovinos, Bos taurus, del distrito Pacanga (La Libertad, Perú). Rebiol 33: 76-83.

9. Espinoza JR, Terashima A, HerreraVelit P, Marcos LA. 2010. Fasciolosis humana y animal en el Perú: impacto en la economía de las zonas endémicas. Rev Perú Med Exp Salud Pública 27: 604-612.

10. Leguía P, Casas E. 1999. Enfermedades parasitarias y atlas parasitológico de camélidos sudamericanos. Lima: Ed de Mar. $190 \mathrm{p}$.

11. López-Lemes M, Hernández S, Acuña AM, Nari A. 2005. Fascioliasis en la República Oriental del Uruguay. Rev Med Uruguay 12: 37-43.

12. Manrique MJ, Cuadros CS. 2002. Fasciolosis: Buscando estrategia de control. Arequipa, Perú: Akuarella. 126 p.

13. Olaechea FV. 2004. Fasciola hepatica. Red de Helmintología de FAO para América Latina y el Caribe. Conferencia electrónica. [Internet]. Disponible en: http://helminto.inta.gob.ar/ Fasciola/FASCIOLA\%-20HEPATICA\%-20Fermin\%20Olaechea.pdf

14. Oliveira MCS, Alencar MM, Chagas ACS, Giglioti R, Oliveira HN. 2009. Gastrointestinal nematode infection in beef cattle of different genetic groups in
Brazil. Vet Parasitol 166: 249-254. doi: 10.1016/j.vetpar.2009.09.006

15. Paredes CP. 2014. Incidencia parasitaria gastrointestinal en la ganadería lechera en la hacienda «Monte Carmelo» sector Urbina provincia Chimborazo. Tesis de Licenciatura. Ambato: Univ. Técnica de Ambato $89 \mathrm{p}$.

16. Paucar SS, Chávez AV, Casas EA, Suárez FA. 2010. Prevalencia de fascioliasis y paramfistomiasis en el ganado lechero de Oxapampa, Pasco. Rev Inv Vet Perú 21: 87-92. doi: 10.15381/ rivep.v21i1.314

17. Prepelitchi L. 2009. Ecoepidemiología de Fasciola hepatica (Trematoda, Digenea) en el norte de la provincia de Corrientes destacando aspectos ecológicos de Lymnaea columella (Pulmo-nata, Lymnaeidae) y su rol como hospe-dador intermediario. Tesis Doctoral. Buenos Aires: Univ. de Buenos Aires. $185 \mathrm{p}$.

18. Rodríguez-Vivas RI. Rodríguez-Vivas LA, Cob-Galera JL, DomínguezAlpizar. 2001. Frecuencia de parásitos gastrointestinales en animales domésticos diagnosticados en Yucatán, México. Rev Biomed 12: 19-25.

19. Rojas M, Torrel T. 2004. Paranfistomidosis. En: Nosoparasitosis de los rumiantes domésticos. Lima: Martegraf. $146 \mathrm{p}$.

20. Sanchis JL, Miguelez S, Solari MA, Pineiro P, Macchi MI, Maldini GI, Venzal JL, et al. 2011. Seroprevalencia de la fasciolosis bovina en el departamento de Salto (Uruguay). Rev IberoLatinoam Parasitol 70: 163-171.

21. Terashima A, Marcos L, Maco V, Canales M, Samalvides F, Tello R. 2009. Técnica de sedimentación en tubo de alta sensibilidad para el diagnóstico de parásitos intestinales. Rev Gastroenterol Perú 29: 305-310.

22. Ticona S, Chávez V, Casas V, Chavera C, Li E. 2010. Prevalencia de Fasciola hepatica en bovinos y ovinos de Vilcashuamán, Ayacucho. Rev Inv Vet Perú 21: 168-174. 
23. Torgerson PR. 2013. One world health: Socioeconomic burden and parasitic disease control priorities. Vet Parasitol 195: 223-232. doi: 10.1016/j.vetpar.2013.-04.004

24. Wilches C, Jaramillo JG, Muñoz DL. 2009. Presencia de infestación por Fasciola hepatica en habitantes del valle de San Nicolás, oriente antioqueño. Infectio 13: 92-99.

25. Zárate R. 2003. Parásitos en rumiantes. En: Memoria II Simposio sobre enfermedades que afectan a los bovinos en el sistema vaca/becerro. Nueva León, México. 